Article

\title{
Quasi-Transient Calculation of Czochralski Growth of Ge Crystals Using the Software Elmer
}

\author{
Wolfram Miller*, Nikolay Abrosimov, Jörg Fischer, Alexander Gybin, Uta Juda, Stefan Kayser \\ and Jószef Janicskó-Csáthy
}

Leibniz-Institut für Kristallzüchtung (IKZ), Max-Born-Str. 2, 12489 Berlin, Germany; nikolay.abrosimov@ikz-berlin.de (N.A.); joerg.fischer@ikz-berlin.de (J.F.); alexander.gybin@ikz-berlin.de (A.G.); uta.juda@ikz-berlin.de (U.J.); stefan.kayser@ikz-berlin.de (S.K.); jozsef.janicsko-csathy@ikz-berlin.de (J.J.-C.)

* Correspondence: wolfram.miller@ikz-berlin.de

Received: 11 November 2019; Accepted: 19 December 2019; Published: 31 December 2019

check for updates

\begin{abstract}
A numerical scheme was developed to compute the thermal and stress fields of the Czochralski process in a quasi-time dependent mode. The growth velocity was computed from the geometrical changes in melt and crystal due to pulling for every stage, for which the thermal and stress fields were computed by using the open source software Elmer. The method was applied to the Czochralski growth of Ge crystals by inductive heating. From a series of growth experiments, we chose one as a reference to check the validity of the scheme with respect to this Czochralski process. A good agreement both for the shapes of the melt/crystal interface at various time steps and the change in power consumption with process time was observed.
\end{abstract}

Keywords: global simulation; Czochralski; germanium

\section{Introduction}

In contrast to silicon, it is still a challenge to grow germanium crystals without dislocations. In specific cases, it is even not wanted to grow dislocation free, but to keep the dislocation density in a certain range. This applies for Ge used in radiation detectors, where the Ge crystal is grown in hydrogen atmosphere and not in argon because of purity reasons. In dislocation-free crystals, a hydrogen double vacancy complex will be formed with hydrogen, which leads eventually to the degradation of the detector [1]. Dislocation multiplication is driven by thermal stresses, and thus, tuning the thermal field in the crystal is of severe importance. In the case of Czochralski growth with resistance heating, numerical results have been presented by Artemyev et al. using the software package CGsim (http://str-soft.com/products/CGSim/) and compared with experimental results [2]. In the case of inductive heating, which is required to grow Ge crystals for detectors, the tuning of the thermal field is much more challenging. Experimental work has been done by Wang et al. [3]. However, no calculations have been made for these growth experiments. A global simulation for the Czochralski growth of Ge with induction heating was performed by Honarmandnia et al. [4]. However, only the temperature field including the interface shape was computed for three distinctive growth stages. Later, they also considered thermal stress, but for a configuration with a resistance heater [5].

In order to obtain information on dislocation density development, it is essential to simulate the entire growth process. This can be done in one run solving the time dependent heat transport equation. This approach is, e.g., realized in CGsim, but with some restrictions on the gas phase attached to the moving parts such as the crystal and melt. Furthermore, the grid is adapted in the parts of changing geometry by condensing and expanding the nodes, leading to a very dense mesh in the shrinking melt volume and a very coarse mesh in the growing crystal. Other approaches include a recalculation of the mesh in the relevant regions during the run as, e.g., that of Sabanski et al. [6]. In this paper, 
we present an approach with different runs for every stage of the process. The crystal shape is preset either by the shape obtained in the experiment or an artificial one. Thus, the three phase junction (melt/gas/crystal) is defined by input, but the melt/crystal interface is calculated. We developed a script for an automatic setup of geometry and meshing for each growth stage. The calculation of the thermal and stress fields using the open source software Elmer (https://www.csc.fi/web/elmer) is started from a script. The shape of the crystal was given previously. In this paper, we use the shapes obtained by the growth experiments to compare experimental and numerical results. However, any artificial shape can be given as an input for the computation.

\section{Growth Experiment and Characterization}

For the Czochralski growth of high purity Ge single crystals, a growth furnace was constructed at IKZ. Details were described elsewhere [7]. A sketch for the axisymmetric calculation is presented in Figure 1. The current setup with a quartz crucible of a $90 \mathrm{~mm}$ inner diameter was used to grow 2 " crystals. The only graphite part in the setup was the susceptor holding the crucible. Heat was generated in the graphite part by inductive heating using a coil with 9 windings made of silver. A frequency of $80 \mathrm{kHz}$ was applied. Crystals were pulled with a constant pulling velocity of $v_{\text {pull }}=0.5 \mathrm{~mm} / \mathrm{min}$, except when the bottom cone was grown to empty the crucible. The position of the crucible was fixed during the entire process of crystal growth. As a result, the melt level was decreasing in time, resulting in a higher growth velocity of the crystal than the pulling velocity. The rotation rates of the crystal and crucible were $15 \mathrm{rpm}$ and $-2 \mathrm{rpm}$, respectively (counter rotation). The radius of the crystal was controlled by adapting the heating power. This was done manually. In the finishing process, the Ge melt was pulled out completely.

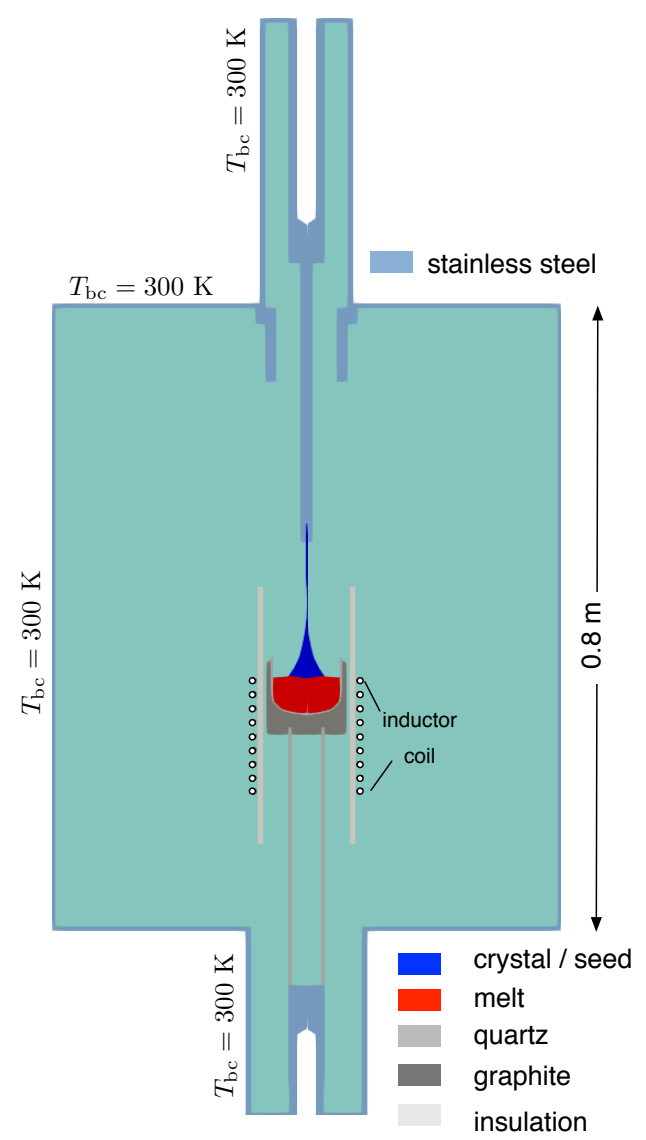

Figure 1. Sketch of the furnace as used for the axisymmetric calculations. At the inner part of the coil (inner side of the black rings), a boundary condition of $T_{\text {coilbc }}=300 \mathrm{~K}$ was set because of the water cooling inside the coil. 
Two kinds of material analyses are relevant in this paper: lateral photovoltaic scanning (LPS) measurements to obtain information on the interface shape during crystal growth [8] and etch pit density (EPD) measurements. Therefore, the crystals were cut orthogonal to the crystal growth direction for counting EPD and parallel for LPS. For EPD analysis, the polished wafers were etched to reveal the dislocations. The etch pit density was determined by manually counting the pits using a light microscope. For better comparability, all wafers were analyzed at the same positions (middle, half radius, edge) along a $\langle 100\rangle$ and a $\langle 110\rangle$ direction. A typical LPS setup was shown in $[9,10]$. Here, a modulated and focused laser beam exited electrons and holes, which drifted/diffused inside the sample. If a resulting doping inhomogeneity existed in the area of electron/hole movement, a potential difference would be detectable between the ohmic rim contacts by using a lock-in-technique. In the case of high charge carrier lifetime and a resulting large mean free path of charge carriers, LPS measurements are quite challenging, because the information of the local resistivity gradient is smeared out. This makes the LPS measurements for high purity Ge extremely difficult. In addition, the high intrinsic charge carrier density at room temperature sets a barrier to the measurability. In order to measure differences in local resistivity, the charge carrier concentration had to be larger than the intrinsic charge carrier density. In the current setup, the intrinsic charge carrier density was about $n_{i}=3 \times 10^{13} \mathrm{~cm}^{-3}$ (at $300 \mathrm{~K}$ ) [11], which corresponded to a resistivity of $\rho_{i}=50 \Omega \mathrm{cm}$. The resistivity of the crystal investigated in this paper was just below this critical value $(\approx 40 \Omega \mathrm{cm})$.

\section{Numerical Solution Strategy}

We wanted to compute the thermal and stress fields in the crystal by an axisymmetric global calculation for a sequence of process times. This should be done automatically considering the changes in geometry and in the growth velocity of the crystal because the melt height was changing during the process. Therefore, we developed a perl script calling to Gmsh (http://gmsh.info/) [12] for geometry and mesh creation and afterwards to Elmer (https://www.csc.fi/web/elmer) for the calculation. Results were analyzed using Paraview (https://www.paraview.org/).

The calculations were performed in steps $\Delta l$ of the crystal length. For all computations presented in this paper, we set $\Delta l=1 \mathrm{~mm}$. Because the pulling velocity was constant, but the melt height was changing, this meant that the time for increasing the crystal length by $\Delta l$ depended on the change in crystal volume and correspondingly in melt volume during this time step. We named the iteration step $t_{\text {it }}$ so that the crystal had a length of $l=\Delta l t_{\text {it }}$ after time step $t_{\text {it }}$. For every $t_{\text {it }}$, we determined the meniscus, the melt height, and the time $\Delta t\left(t_{\mathrm{it}}\right)$ required for the growth from $l\left(t_{\mathrm{it}}-1\right)=\Delta l\left(t_{\mathrm{it}}-1\right)$ to $l\left(t_{\mathrm{it}}\right)=\Delta l t_{\mathrm{it}}$. The crystal shape was given by a data file originating either from the shape of the crystal grown or a given profile. The input data points were used for the definition of a spline function representing the shape of the crystal. Thus, we had a smooth function without abrupt changes in the boundary of the crystal. Therefore, the change in the growth angle was also continuous, which was important for the computation of the meniscus. The meniscus height $h_{\text {menis }}$ was computed by the approach of Hurle [13]:

$$
h_{\text {menis }}=a_{L}\left[\frac{1.0-\cos \left(\alpha_{\mathrm{gr}}\right)}{1.0+a_{L} /\left(\sqrt{2} R_{X}\right)}\right]^{1 / 2} .
$$

The definitions are given in Figure 2. In particular, we have $\alpha_{\mathrm{gr}}=\pi / 2-\left(\alpha_{\text {wet }}+\alpha_{\mathrm{X}}\right)$, where $\alpha_{\mathrm{X}}$ is obtained by:

$$
\alpha_{X}=\operatorname{atan}\left(\frac{R_{X}\left(t_{\mathrm{it}}\right)-R_{X}\left(t_{\mathrm{it}-1}\right)}{\Delta l}\right)
$$

$a_{L}$ is the Laplace constant, defined as $a_{L}=\sqrt{2 \gamma_{\mathrm{lg}} /\left(\rho_{m} g\right)}$, where $\gamma_{\mathrm{lg}}$ is the surface tension between the melt and gas. $\rho_{m}, \Delta H$, and $g$ are the density of the melt, latent heat, and gravitational acceleration, respectively. In order to obtain the melt-gas interface, we computed the height $h$ above the melt level at the crucible. Together with the corresponding radius $r$, we could computed a set of points of the melt-gas interface: 


$$
\begin{aligned}
h & =a_{L}\left[\frac{1.0-\sin (\alpha)}{1.0+a_{L} /(\sqrt{2} r)}\right]^{1 / 2}, \\
r & =\frac{\sqrt{2} R_{X}}{a_{L}} \ln \left(\frac{\tan \left(\frac{1}{4} \alpha_{\mathrm{gr}}\right)}{\tan \left(\frac{1}{4} \alpha\right)}\right)+2\left(\cos \left(\frac{1}{2} \alpha\right)-\cos \left(\frac{1}{2} \alpha_{\mathrm{gr}}\right)\right) .
\end{aligned}
$$

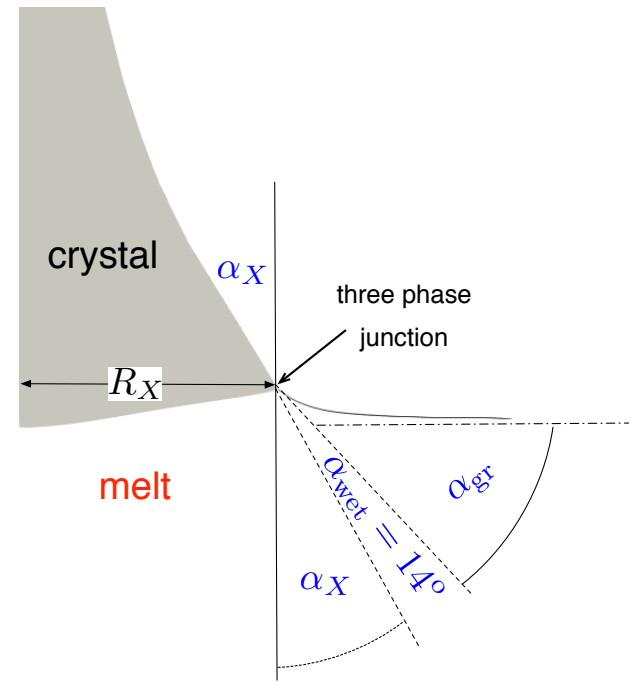

Figure 2. Definitions of the angles for meniscus calculations. The wetting angle is $\alpha_{\text {wet }}=14^{\circ}$ [14].

The calculation was done for values of $\alpha$ between $0^{\circ}$ and $\alpha_{\mathrm{gr}}$ in steps of $5^{\circ}$. For every iteration $t_{\mathrm{it}}$, the crystal volume could be computed, and thus, the required melt volume was known. The latter was used to compute the melt level at the crucible $h_{\mathrm{mc}}$ by a simple numerical integration taking into account the melt volume beneath the crystal because of the meniscus $\left(V=\pi R_{X}^{2} h_{\text {menis }}\right)$. The melt volume below the meniscus itself was not taken into account. Getting $h_{\text {total }}=h_{\mathrm{mc}}+h_{\text {menis }}$, we can now compute the time step as:

$$
\Delta t=\left(\Delta l+h_{\text {total }}(i t)-h_{\text {total }}(i t-1)\right) / v_{\text {pull }}
$$

and the growth velocity by:

$$
v_{n}=\Delta l / \Delta t .
$$

This velocity was used for setting the latent heat production at the interface. At this point, we would like to note that the shape of the crystal was an input for the calculations. The shape might be taken as the crystal grown in an experiment or one can preset an artificial shape. Therefore, the three phase junction (melt/gas/crystal) was defined by the input and not an object to be computed. However, one obtained the required input power as a function of time, which could be adapted in an experiment. In this paper, we used the shapes obtained in the experiments. The shape was defined via the picture of the crystal and normalized by a measured radius at a specific height.

The computation of the thermal field included heat conduction and wall-to-wall radiation, where the parts made of quartz were treated as transparent without refraction. Computations with and without gas convection both for argon and hydrogen showed that there was no significant change of the temperature field in the crystal and only little in power. In the quasi-transparent calculation, we neglected the effect of the moving crystal for the heat exchange. For the small pulling rates like $0.5 \mathrm{~mm} / \mathrm{min}$ or $1.0 \mathrm{~mm} / \mathrm{min}$ and the crystal radius of about $2^{\prime \prime}$, this effect was small. In the melt, we additionally computed the advective heat transport. We took into account the forced convection induced by crucible and crystal rotation, as well as the Marangoni convection at the melt/gas interface and the buoyancy convection in the volume. Melt convection was always the critical point in such calculations. In order to obtain a stationary solution, either the turbulence model or an increased viscosity could be used. For a realistic time dependent solution, a 3D calculation was required, which 
meant a much higher computational effort. Here, we solved the time independent Navier-Stokes equations directly without employing a turbulence model. Therefore, the viscosity used in the computation was larger by about a magnitude than the real one. In the next section, we will show that the agreement in the interface shapes during growth was good between experiment and numerical simulation, i.e., the heat transport in the melt was described well by the convection with the increased viscosity. In order to get a stable solution, we started with a viscosity of $\mu=2 \times 10^{-2}$ Pa s, which decreased linearly over 20 inner iterations to $\mu=5 \times 10^{-3}$ Pa s.

At the outer boundary of the furnace, we set a constant temperature of $T_{\mathrm{bc}}=300 \mathrm{~K}$. We also set this temperature at the inner boundary of the inductor coil $\left(T_{\text {coilbc }}=300 \mathrm{~K}\right)$; the coil was water cooled.

The power was adjusted by setting the melting point temperature of Ge $(1210 \mathrm{~K})$ at the three phase line as the target value for a feedback loop. The isoline of melting point temperature was assumed to be the melt/crystal interface. The actual interface in an iteration was moved according to the distance from the isoline of melting point temperature. The mesh in crystal and melt was adapted by solving the equation for elastic deformation. As this solver in Elmer had by default the variable displacementfor the displacement of the nodes, one had to define a new variable (e.g., displacement-stress) for the displacement used in the solver for the thermal stress.

The elastic stress due to the thermal strain $\epsilon_{T}=\alpha_{L}\left(T-T_{\text {ref }}\right)$ with $T_{\text {ref }}=595 \mathrm{~K}$ was computed. $\alpha_{L}$ is the thermal expansion coefficient, and the value is given in Table 1 . We used the temperature dependent elastic coefficients as given in Table 2 and employed the approach of Lambropoulos [15] for a crystal growing in the $\langle 001\rangle$ direction:

$$
\left(\begin{array}{c}
\sigma_{r r} \\
\sigma_{\phi \phi} \\
\sigma_{z z} \\
\sigma_{r z}
\end{array}\right)=\left(\begin{array}{cccc}
C_{11}+\frac{1}{4} H & C_{12}-\frac{1}{4} H & C_{12} & 0 \\
C_{12}-\frac{1}{4} H & C_{11}+\frac{1}{4} H & C_{12} & 0 \\
C_{12} & C_{12} & C_{11} & 0 \\
0 & 0 & 0 & C_{44}
\end{array}\right)\left(\begin{array}{c}
\epsilon_{r r}-\epsilon_{T} \\
\epsilon_{\phi \phi}-\epsilon_{T} \\
\epsilon_{z z}-\epsilon_{T} \\
\epsilon_{r z}
\end{array}\right)
$$

where $H$ is given by $H=2 C_{44}+C_{12}-C_{11}$. The stress calculation was performed in the seed and crystal. Displacements in the vertical direction were set to zero at the top of the seed.

Table 1. Physical properties of liquid and solid Germanium as used for the calculations.

\begin{tabular}{|c|c|c|c|c|}
\hline Ge Melt & & & & \\
\hline Density & $\rho_{m}$ & $\mathrm{~kg} / \mathrm{m}^{3}$ & 5534 & * \\
\hline Heat capacity & $c_{p}$ & $\mathrm{Jkg}^{-1} \mathrm{~K}^{-1}$ & $358\left(@ T_{m}\right)$ & [16] \\
\hline Thermal expansion & $\alpha_{\mathrm{V}}$ & $\mathrm{K}^{-1}$ & $(1.06 \pm 0.02) \times 10^{-4}$ & [17] \\
\hline Heat conductivity & $\lambda$ & $\mathrm{Wm}^{-1} \mathrm{~K}^{-1}$ & $48\left(@ T_{m}\right)$ & [18] \\
\hline Emissivity & $\varepsilon$ & & 0.2 & [19] \\
\hline Electrical conductivity & $\sigma_{\mathrm{el}}$ & $\mathrm{S} / \mathrm{m}$ & $1.4 \times 10^{6}$ & [20] \\
\hline Dynamic viscosity & $\mu$ & Pas & $6.8 \times 10^{-4}\left(@ T_{m}\right)$ & [21] \\
\hline Prandtl number & $\operatorname{Pr}$ & & 0.05 & \\
\hline Marangoni coefficient & & $\mathrm{Nm}^{-1} \mathrm{~K}^{-1}$ & $7.32 \times 10^{-5}$ & [22] \\
\hline Latent heat & $\Delta H$ & $\mathrm{~J} / \mathrm{kg}$ & $4.65 \times 10^{5}$ & [14] \\
\hline Laplace constant & $a_{L}$ & $\mathrm{~m}$ & $5.3 \times 10^{-3}$ & \\
\hline \multicolumn{5}{|l|}{ Ge Solid } \\
\hline Density & $\rho_{s}$ & $\mathrm{~kg} / \mathrm{m}^{3}$ & 5370 (@300K) & [23] \\
\hline Heat capacity & $c_{p}$ & $\mathrm{Jkg}^{-1} \mathrm{~K}^{-1}$ & 418 & [14] \\
\hline Heat conductivity & $\lambda$ & $\mathrm{Wm}^{-1} \mathrm{~K}^{-1}$ & $\approx 17(800-1200 \mathrm{~K})$ & [24] \\
\hline Emissivity & & $\varepsilon$ & 0.55 & [25] \\
\hline thermal expansion & $\alpha_{\mathrm{L}}$ & $\mathrm{K}^{-1}$ & $7.5 \times 10^{-6}$ & [23] \\
\hline \multirow[t]{3}{*}{ Elastic constants } & $C_{11}$ & $\mathrm{~Pa}$ & $13.2 \times 10^{10}-1.7 \times 10^{7} \mathrm{~K}^{-1} \mathrm{~T}$ & [26] \\
\hline & $C_{12}$ & $\mathrm{~Pa}$ & $2.8 \times 10^{10}-8.2 \times 10^{6} \mathrm{~K}^{-1} \mathrm{~T}$ & [26] \\
\hline & $C_{44}$ & $\mathrm{~Pa}$ & $5.2 \times 10^{10}-5.4 \times 10^{6} \mathrm{~K}^{-1} \mathrm{~T}$ & [26] \\
\hline
\end{tabular}

${ }^{*}$ Intermediate value between the measurements of $[16,21]$. 
Table 2. Physical properties of furnace materials.

\begin{tabular}{|c|c|c|}
\hline Stainless Steel & & \\
\hline Density & $\rho$ & $7000 \mathrm{~kg} / \mathrm{m}^{3}$ \\
\hline Heat capacity & $c_{p}$ & $570 \mathrm{Jkg}^{-1} \mathrm{~K}^{-1}$ \\
\hline Heat conductivity & $\lambda$ & $1.5 \mathrm{Wm}^{-1} \mathrm{~K}^{-1}$ \\
\hline Emissivity & $\varepsilon$ & 0.1 \\
\hline Electrical conductivity & $\sigma_{\mathrm{el}}$ & $1.4 \times 10^{6} \mathrm{~S} / \mathrm{m}$ \\
\hline \multicolumn{3}{|l|}{ Graphite $^{\dagger}$} \\
\hline Density & $\rho$ & $1750 \mathrm{~kg} / \mathrm{m}^{3}$ \\
\hline Heat capacity & $c_{p}$ & $927 \mathrm{Jkg}^{-1} \mathrm{~K}^{-1}$ \\
\hline Heat conductivity & $\lambda$ & $90 \mathrm{Wm}^{-1} \mathrm{~K}^{-1}$ \\
\hline Emissivity & $\varepsilon$ & 0.8 \\
\hline Electrical conductivity & $\sigma_{\mathrm{el}}$ & $2.0 \times 10^{5} \mathrm{~S} / \mathrm{m}$ \\
\hline \multicolumn{3}{|l|}{ Quartz $\ddagger$} \\
\hline Density & $\rho$ & $2200 \mathrm{~kg} / \mathrm{m}^{3}$ \\
\hline Heat capacity & $c_{p}$ & $1000 \mathrm{Jkg}^{-1} \mathrm{~K}^{-1}$ \\
\hline Heat conductivity & $\lambda$ & $\left(1.8-1.21 \times 10^{-3} \mathrm{~K}^{-1} \mathrm{~T}+1.75 \times 10^{-6} \mathrm{~K}^{-2} \mathrm{~T}^{2}\right) \mathrm{Wm}^{-1} \mathrm{~K}^{-1}$ \\
\hline Electrical conductivity & $\sigma_{\mathrm{el}}$ & $0 \mathrm{~S} / \mathrm{m}$ \\
\hline \multicolumn{3}{|l|}{ Heat Shield } \\
\hline Density & $\rho$ & $4000 \mathrm{~kg} / \mathrm{m}^{3}$ \\
\hline Heat capacity & $c_{p}$ & $680 \mathrm{Jkg}^{-1} \mathrm{~K}^{-1}$ \\
\hline Heat conductivity & $\lambda$ & $3.3 \mathrm{Wm}^{-1} \mathrm{~K}^{-1}$ \\
\hline Emissivity & $\varepsilon$ & 0.2 \\
\hline Electrical conductivity & $\sigma_{\mathrm{el}}$ & $0 \mathrm{~S} / \mathrm{m}$ \\
\hline
\end{tabular}

${ }^{\dagger}$ Values of EK98300 K. ${ }^{\ddagger}$ Data from the data sheets of Heraeus.

Calculations were performed on a typical desktop computer. For the crystals considered in this paper and $\Delta l=1 \mathrm{~mm}$, the complete calculation took about one day.

\section{Results and Discussion}

We considered two experimental runs for our numerical simulations. The examples were chosen out of a series of growth experiments for the process development, which was started with argon as the atmosphere and was later changed to hydrogen. In one case, we grew a crystal of $513 \mathrm{~g}$ total weight in an argon atmosphere (Case 1) and in the other one a crystal of $1530 \mathrm{~g}$ total weight in a hydrogen atmosphere (Case 2). Firstly, we discuss the results of Case 2. The crystal shape of the final crystal can be seen on the left hand side of Figure 3. Three wafers were cut out of the crystal as indicated by the horizontal yellow lines. These wafers were used for the measurements of the etch pitch density (EPD), which we will discuss later. Three vertical cuts were made, in the cone, the cylindrical part, and the final cone. All three were polished chemo-mechanically and used for LPS measurements. In the middle of Figure 3, the outcome of LPS is shown together with the computed interface lines (yellow). A good agreement can be observed over the entire growth process. Some minor differences can be seen in the middle of the shouldering. For comparison, on the right hand side, the interface lines are presented, which were obtained by running the calculation using $v_{\text {pull }}$ for computing the latent heat as was done in the standard computational scheme $\left(v_{n} \equiv v_{\text {pull }}\right)$. A significant difference in the shapes can be observed in the later stages of the growth, especially towards the end. The shape of the interface is of major importance for the von Mises stress in its vicinity. In Figure 4, the von Mises stress is shown for three different locations at the interface for the two runs with computed $v_{n}$ and with $v_{n} \equiv v_{\text {pull }}$. The stress was always higher for $v_{n} \equiv v_{\text {pull }}$ except for a short time period. Because of the high temperature, the von Mises stress in the vicinity of the interface was the most critical for the multiplication of dislocations. 


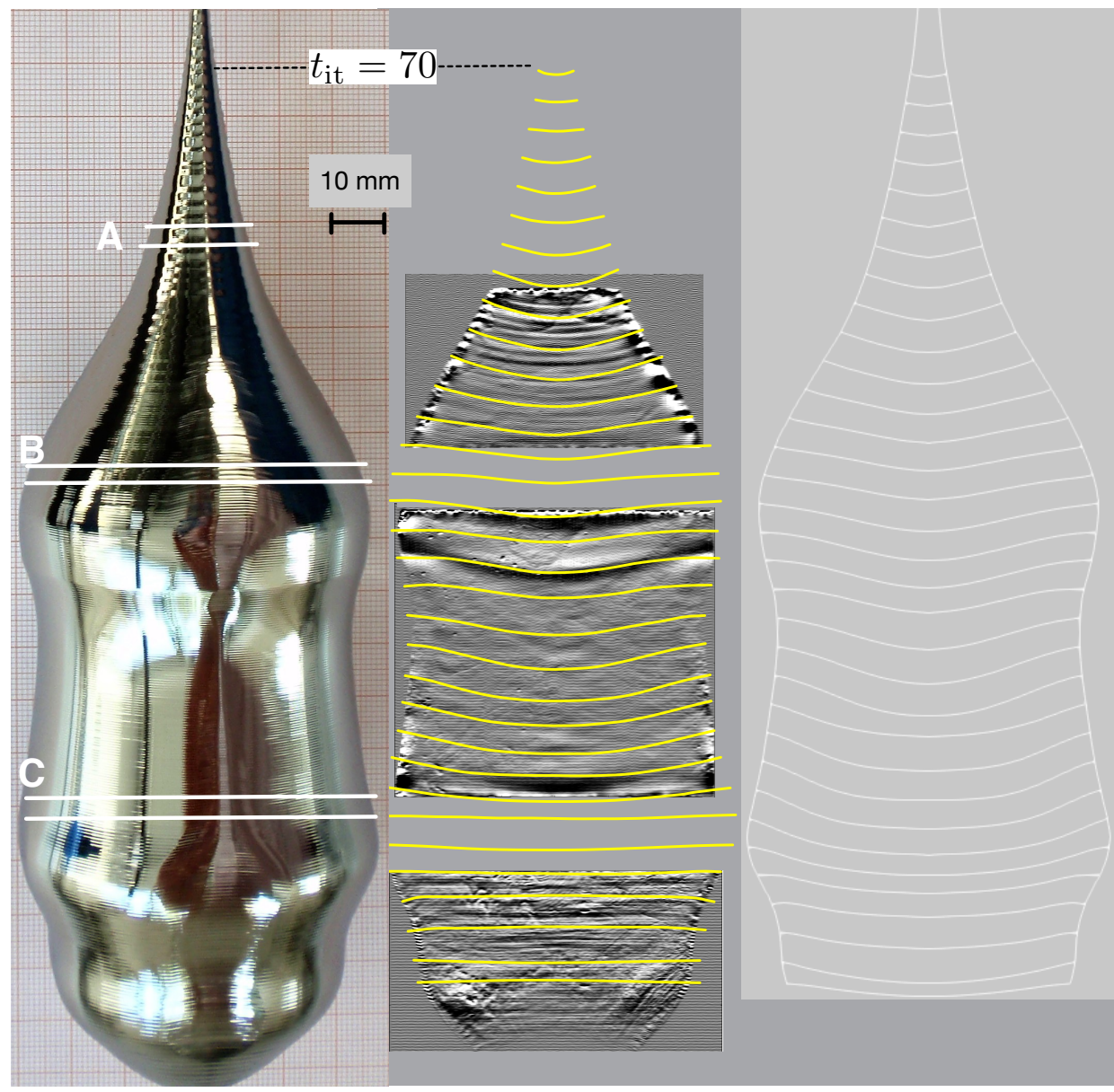

Figure 3. Left: Crystal grown (Case 2) with the indication of horizontal cutting planes for etch pit density (EPD) measurements (A,B,C). Middle: Lateral photovoltaic scanning (LPS) at three vertical cuts and interface lines (yellow) obtained in the numerical calculation. Right: Interface lines for calculation with $v_{n} \equiv v_{\text {pull }}$.

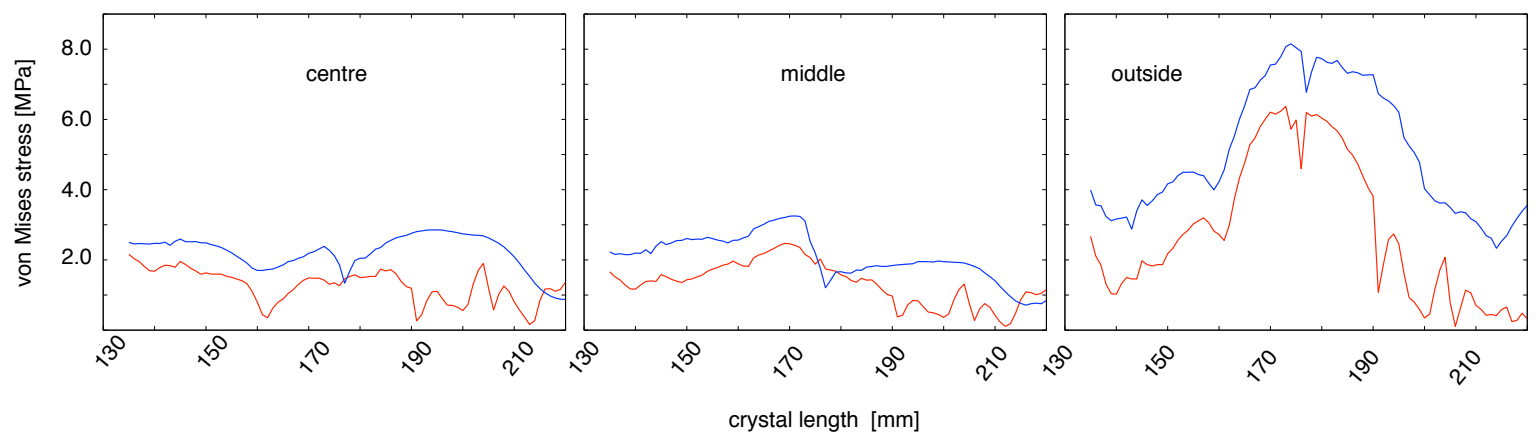

Figure 4. Average von Mises stress at the interface as a function of the growth stage (crystal length) for Case 2. Left: At the centre At the centre $(r=0-3 \mathrm{~mm})$; middle: around $R_{X} / 2(r=10-13 \mathrm{~mm})$; right: near the rim $(r=18-21 \mathrm{~mm})$. The lower red curve is for the case with computed $v_{n}$ and upper blue one for the case with $v_{n} \equiv v_{\text {pull }}$.

We also compared the change in the required power during the process time. Please note that the experimental value was given by the electrical current and voltage in the entire circuit, whereas the numerical values were the sum of the induced heat in the graphite and melt. For comparison, we shifted the experimental values by $350 \mathrm{~W}$. The comparison is shown in Figure 5. After shouldering, the required power went down. There was some instability when reaching the cylindrical part, which 
was more pronounced in the numerical calculation than in the experiment. This might be because we did not perform fully transient calculations and did not take the change of the interface shape into account for the computation of $v_{n}$. The growth velocity was computed before the thermal calculation with Elmer starts and was not adapted later. This meant that the latent heat production corresponded to the evolution of a flat interface. Latent heat effects due to changes in the interface shape were not considered. The second instability occurred near the end of the process. For a better comparison, the crystal radius is also plotted in Figure 5. Please note that the plot was versus process time and not versus crystal length. Therefore, the shape given by the plot of the crystal radius differed from that of the crystal shape. We also show the result if only the change in the melt level was taken into account for computing $v_{n}$ and the change of the meniscus height was neglected (light orange curve). In the regions where the crystal radius was going from an increasing to a decreasing one, a strong peak in power occurred. In these regions, the large change of $\alpha_{\mathrm{gr}}$ led to a significant change of $h_{\text {menis }}$ (see Equation (1) and the melt volume beneath the crystal. Therefore, taking into account the effect of this melt volume in the computation of the growth velocity had a strong impact on the power consumption.

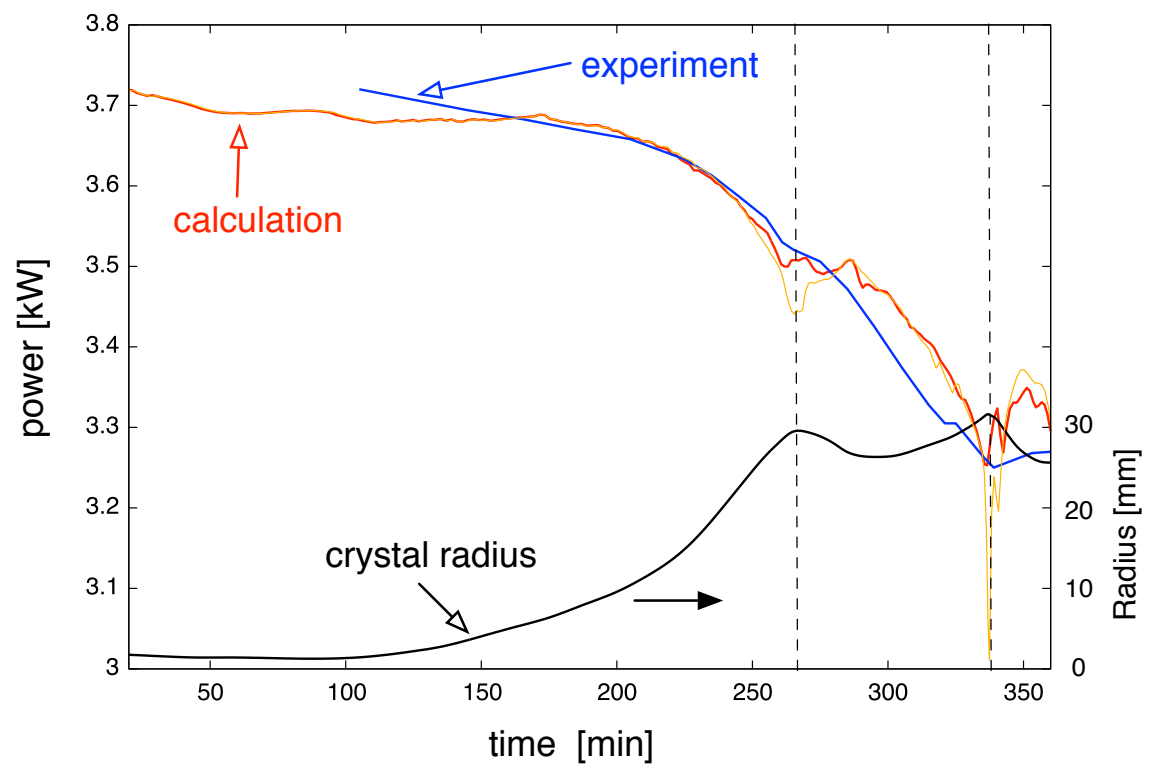

Figure 5. Power as a function of the process time for Case 2. The calculated power is measured as the sum of induced power in the graphite susceptor and melt. The experimental value is the measured electrical power put into the entire coil system including the resonator circuit. It is reduced by $350 \mathrm{~W}$ to be comparable with computed curve. The light orange curve is for the computation without taking into account the melt volume change due to the change in meniscus height. For an easier analysis, the crystal radius is also given (scale on right hand side).

One of the key process parameters was the pulling velocity. The higher the velocity, the larger the amount of latent heat released and the lower the required power. For Case 2, we computed the process again for a pulling velocity of $v_{\text {pull }}=1 \mathrm{~mm} / \mathrm{min}$. In Figure 6 , we show at the bottom the power consumption as a function of the process time. Please note that for the same length of the crystal, the process time was different for the two cases. The dotted lines indicate the growth stage where the von Mises stress in the crystal and the temperature in the melt are shown on the left hand side. For each stage, the left part and right part show the results for $v_{\text {pull }}=0.5 \mathrm{~mm} / \mathrm{min}$ and $v_{\text {pull }}=1.0 \mathrm{~mm} / \mathrm{min}$, respectively. The reduced power consumption for $v_{\text {pull }}=1 \mathrm{~mm} / \mathrm{min}$ had a strong impact on the temperature in the melt (see Figure 6 top). The melt would freeze at the top in the vicinity of the crucible. This meant that the position of the crucible should be changed when applying a high pulling velocity. The von Mises stress depended significantly on the growth stage and was sometimes larger in one case and sometimes in the other. We would like to note that we took the shape of the crystal as 
observed in the experiment with $v_{\text {pull }}=0.5 \mathrm{~mm} / \mathrm{min}$. Therefore, a general statement on stresses and possible dislocation multiplication cannot be made. However, the situation for the melt/crystal interface was clear: the interface for $v_{\text {pull }}=1.0 \mathrm{~mm} / \mathrm{min}$ was always concave, whereas for $v_{\text {pull }}=0.5 \mathrm{~mm} / \mathrm{min}$, it was always convex. We would like to mention that with the algorithm developed, we could give any crystal shape as an input and check the optimal pulling velocity. As an illustration of how the growth velocity was changing during the growth process in Case 2, we show the $v_{n}$ as a function of the process time in Figure 7 . One can see that the main deviations from the pulling velocity $v_{\text {pull }}=0.5 \mathrm{~mm} / \mathrm{min}$ were in the regions of increasing radius.

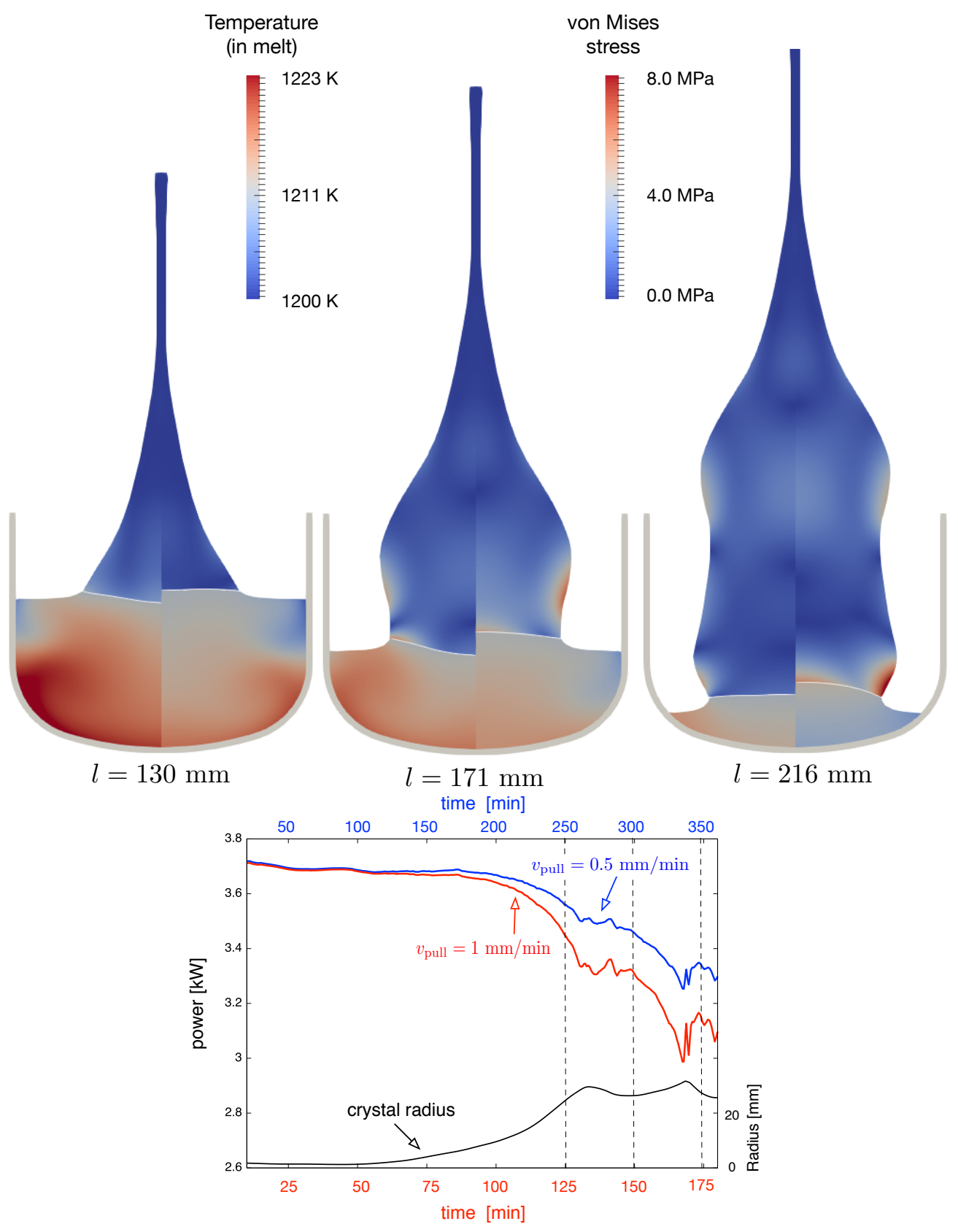

Figure 6. Top: The von Mises stress in the crystal and the temperature in the melt are shown for three different growth stages. Left part of the crystal and melt: $v_{\text {pull }}=0.5 \mathrm{~mm} / \mathrm{min}$. Right part of the crystal and melt: $v_{\text {pull }}=1.0 \mathrm{~mm} / \mathrm{min}$. Bottom: The power consumption for the two runs is plotted as a function of process time. The three stages are indicated by dotted lines. Please note the time scales for $v_{n}=1 \mathrm{~mm} / \mathrm{min}$ at the bottom and for $v_{n}=0.5 \mathrm{~mm} / \mathrm{min}$ at top. 


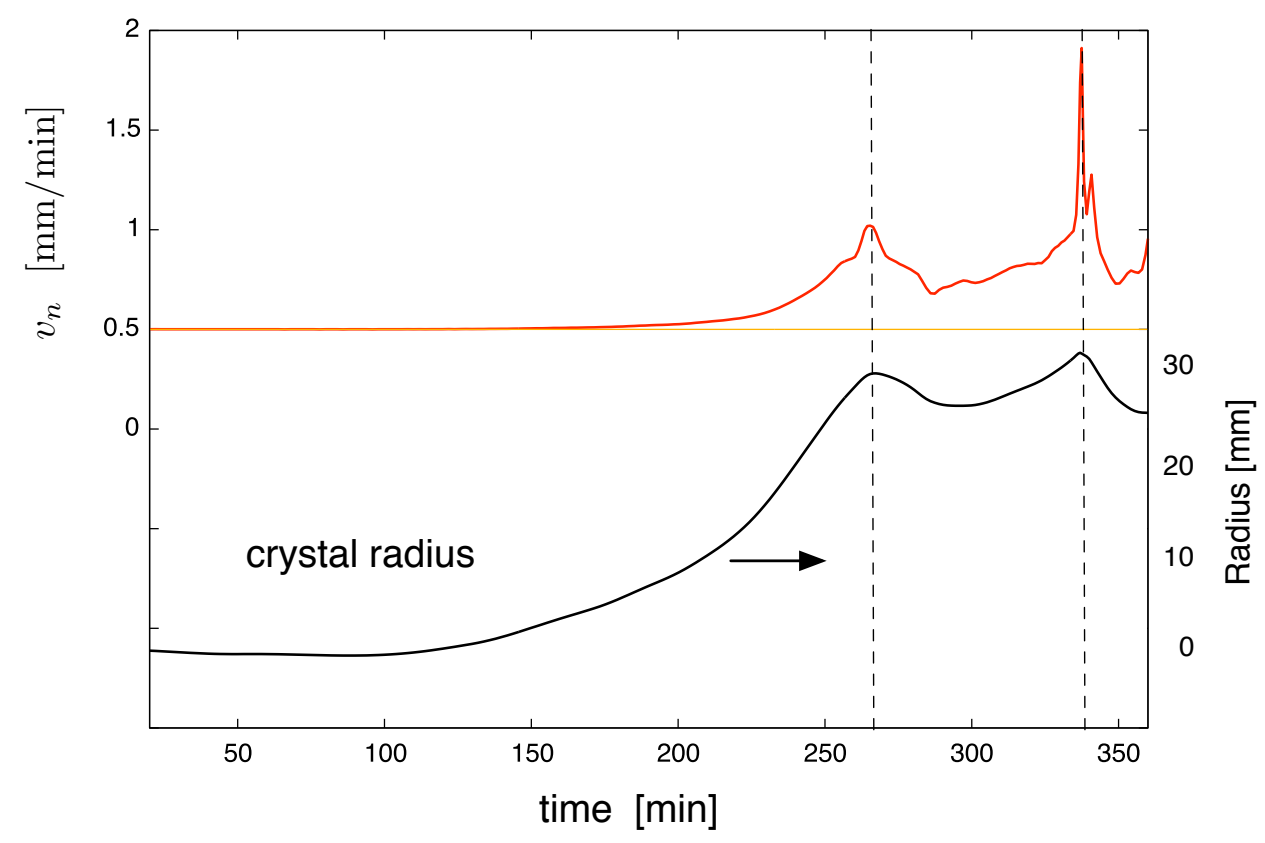

Figure 7. Growth velocity as a function of the process time for Case 2.

A transient or quasi-transient calculation was the prerequisite for computing the dislocation density. However, this was beyond the purpose of this paper, and we only wanted to make some simple calculations to estimate the situation for the two different growth cases. In the Alexander-Haasen model [27], the multiplication of dislocations is given by (see the Nomenclature):

$$
\dot{N}_{\text {disl }}=\underbrace{K k_{0} \tau_{\text {eff }}^{(m+l)} \exp \{-Q / k T\}}_{\mathcal{F}} N_{\text {disl }} .
$$

The effective strain is given by $\tau_{\text {eff }}=\sqrt{J_{2}}-D \sqrt{N_{\text {disl }}}$ with $J_{2}=\frac{1}{2} \sum_{i, j} S_{i j}^{2}$ and $S_{i j}=\sigma_{i j}=\delta_{i j} \frac{1}{3} \sum_{k} \sigma_{k k}$. $D$ is the hardening factor. In this paper, we neglected the hardening effect and set $\tau=\tau_{\text {vonMises, }}$, the von Mises stress. For Ge, $l=1$, and the Peierls energy was $Q=1.6 \mathrm{eV}$ [28]. The same value could be obtained from the data in Figure 10 of [29] when choosing $m=2.24$ for both temperatures. In addition, one obtains $k_{0}=2.6 \times 17^{-12} \mathrm{~m}^{5.48} \mathrm{~N}^{-1} \mathrm{~s}^{-1}$. Taking $K=190 \mathrm{~m} / \mathrm{N}$ [30], we obtain:

$$
\mathcal{F}=4.94 \times 10^{-10} \tau_{\text {eff }}^{3.24} \exp \left\{-1.856 \times 10^{4} / T\right\}
$$

Typically, EPD is measured on three wafers: one in the cone, one at the beginning of the cylindrical part, and one at the end of the cylindrical part. On each wafer, measurements were made at the centre, at $R_{X} / 2$ and $R_{X}$, where $R_{X}$ is the radius of the wafer. Measurements were done in two crystallographic directions $\langle 100\rangle$ and $\langle 110\rangle$ on a $\langle 001\rangle$ oriented plane. Because we performed axisymmetric calculations, we used the average value of the two directions. For the wafer in the cone, we only used one average value because the radius was much smaller than for the wafers from the cylindrical part. The EPD values $N_{\mathrm{EPD}}$ for Cases 1 and 2 are given in Figure 8. For all data points with average values, we also show the maximum variation. EPD was much higher in Case 2 than in Case 1. Please note that in Case 1, argon was used instead of hydrogen, as in Case 2. We also would like to stress that the crystal in Case 2 was grown as a reference crystal, and the process was not optimized with respect to the dislocation densities. In order to make any comparison with our numerical calculations, we summed up the volume averaged multiplication factor $\mathcal{F}$ in a certain region in time:

$$
\mathcal{F}_{S}=\sum_{t} \mathcal{F}\left(t_{\text {it }}\right) \Delta t
$$


These numbers are given in blue in Figure 8. They were significant larger for Case 2 than for Case 1. Thus, the observed tendency in EPD was reflected in the numerical results. The EPD was between 10 and 30 times larger in Case 2 than in Case 1. The calculations exhibited a similar behavior, but the numbers at the centre in Case 2 and those at the rim for Case 1 showed a disagreement from the trend. Nevertheless, the calculation strategy could be already used to find the process conditions where the dislocation density was low. However, a further improvement of the model was required, where the plastic strain should be included in the stress computation (Equation (6)), which was demonstrated by Helbig et al. [31]. In addition, the dislocation density should be computed using Equation (7).
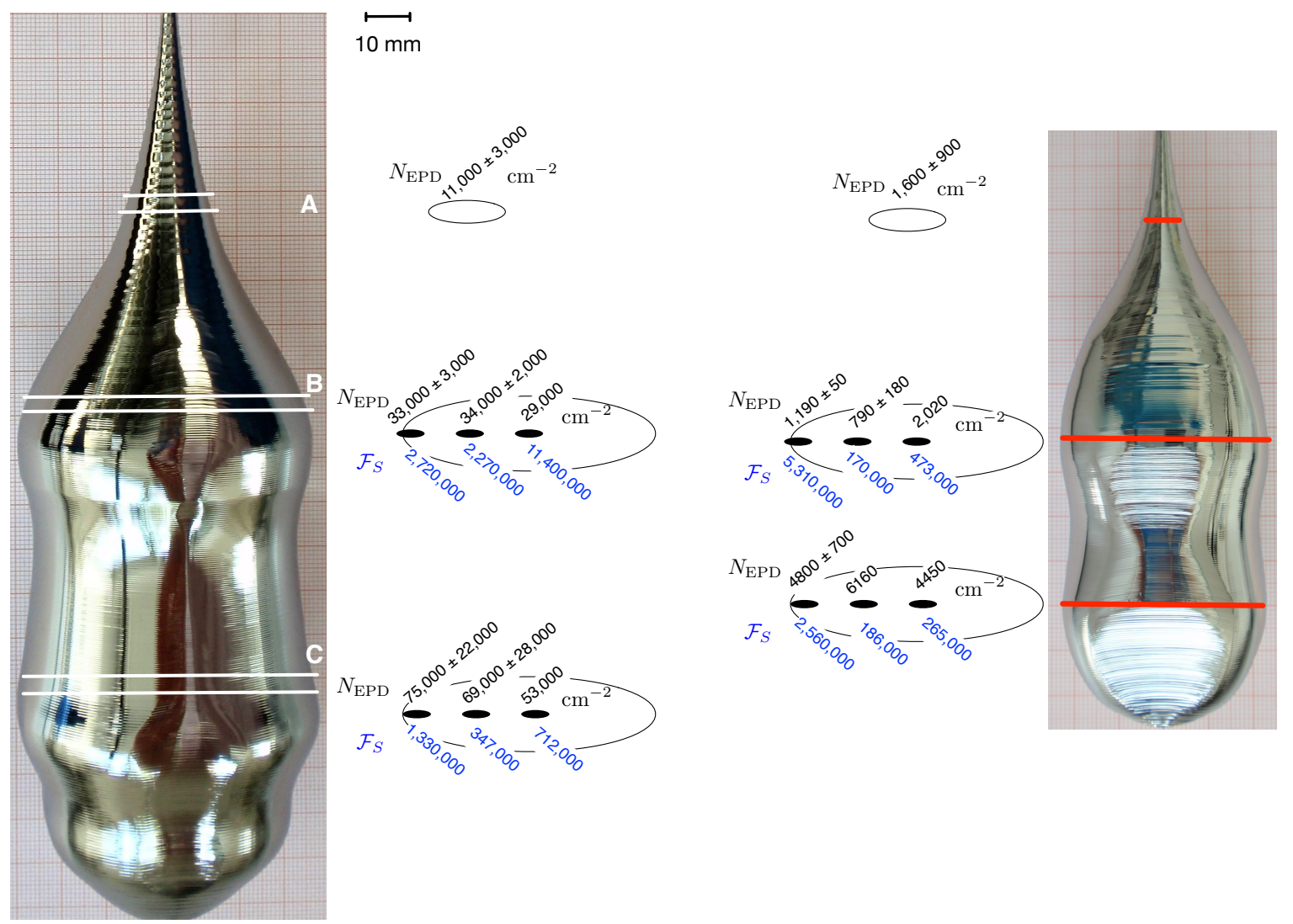

Figure 8. Left: EPD for Case $2\left(N_{\mathrm{EPD}}\right.$ and computed factor for the multiplication of dislocations $\left.\mathcal{F}_{S}\right)$. The wafers A-C correspond to the cutting planes given for the crystal in Figure 3. Right: EPD for Case 1 and computed factor for the multiplication of dislocations. The cutting planes are indicated at the crystals.

\section{Conclusions}

We developed a quasi-transient approach to compute the Czochralski growth of high purity Ge using the open source software Elmer. The approach was validated by a reference experiment. The key point was the comparison of the interface shape during the growth. For this reason, lateral photoscanning (LPS) measurements were performed at vertical cuts through the crystal. A good agreement between LPS measurements and numerical results was observed. We showed that it was essential to compute the local growth velocity instead of using the constant pulling velocity. Furthermore, the change in power with process time was in good agreement between the measured one in the experiment and the calculated one in the numerical calculations.

Employing the temperature field and the von Mises stress, we obtained a first guess for the evolution of the dislocation densities. The tendency from this approach with respect to the growth process and process time was the same as observed by the etch pit densities measured on three wafers 
of a crystal. A complete calculation of dislocation densities applying the Alexander-Haasen model requires a much more advanced numerical approach, which is a project for the future.

Author Contributions: Conceptualization, W.M.; methodology, W.M.; formal analysis, S.K. and U.J.; investigation, A.G., J.F. and J.J.-C.; writing-original draft preparation, W.M., S.K.; writing-review and editing, A.G., U.J. and J.J.-C.; supervision, N.A.; project administration, J.J.-C. All authors have read and agreed to the published version of the manuscript.

Funding: This research was funded by Federal Ministry of Education and Research (BMBF) grant number 05A2017.

Acknowledgments: Part of this work was funded by the Federal Ministry of Education and Research (BMBF) within the Collaborative Research Project (05A2017). We would like to thank F. M. Kießling for his continuous support of the GERDA project.

Conflicts of Interest: The authors declare no conflict of interest.

\section{Nomenclature}

$\Delta l \quad$ change in crystal length in one time iteration step

$\Delta t \quad$ real time of a time iteration step

$a_{L} \quad$ Laplace constant

$b \quad$ Burgers vector

$C_{i j} \quad$ Elastic coefficients

$\mathcal{F} \quad$ Dislocation multiplication factor

$g \quad$ gravitational acceleration

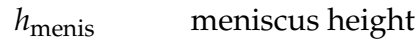

$h_{\mathrm{mc}} \quad$ melt height at the crucible

$h_{\text {total } \quad \text { total melt height }}$

$h \quad$ height of melt/gas interface above the melt at the crucible

$k \quad$ Boltzmann constant

$k_{0} \quad$ material constant for dislocation velocity

$K$ prefactor for dislocation multiplication

$l \quad$ crystal length

$N_{\text {disl dislocation density }}$

$Q \quad$ Peierls energy

$R_{X} \quad$ crystal radius

$r \quad$ radial coordinate of the melt/gas interface

$t_{\text {it }} \quad$ time iteration step

$T_{\mathrm{bc}} \quad$ temperature at the outer boundary

$T_{\text {coilbc }} \quad$ temperature at the inner part of the coils

$T$ temperature

$v_{n} \quad$ growth velocity

$v_{\text {pull }} \quad$ pulling velocity

$\alpha_{X} \quad$ angle of the crystal rim with respect to the vertical direction (see Figure 2)

$\alpha_{\text {wet }} \quad$ wetting angle (see Figure 2)

$\alpha_{\text {gr }} \quad$ growth angle (see Figure 2)

$\epsilon_{i j} \quad$ strain components

$\epsilon_{i j}^{c} \quad$ components of plastic (creep) strain

$\sigma_{i j} \quad$ stress components

$\tau \quad$ stress

$\tau_{\text {eff }} \quad$ effective stress

$\tau_{\text {vonMises }} \quad$ von Mises stress

\section{References}

1. Sande, M.V.; Goethem, L.V.; Laet, L.D.; Guislain, H. Dislocations in high-purity germanium and its relation to $\gamma$-ray detector performance. Appl. Phys. A 1986, 40, 257-261. [CrossRef] 
2. Artemyev, V.V.; Smirnov, A.D.; Kalaev, V.V.; Mamedov, V.M.; Sidko, A.P.; Podkopaev, O.I.; Kravtsova, E.D.; Shimansky, A.F. Modeling of dislocation dynamics in germanium Czochralski growth. J. Cryst. Growth 2017, 468, 443-447. [CrossRef]

3. Wang, G.; Guan, Y.; Mei, H.; Mei, D.; Yang, G.; Govani, J.; Khizar, M. Dislocation density control in high-purity germanium crystal growt. J. Cryst. Growth 2014, 393, 54-58. [CrossRef]

4. Honarmandnia, M.; Tavakolia, M.H.; Sadeghib, H. Global simulation of an rf czochralski furnace during different stages of germanium single crystal growth. CrystEngComm 2016, 18, 3942-3948. [CrossRef]

5. Tavakoli, M.H.; Renani, E.K.; Honarmandnia, M.; Ezheiyan, M. Computational analysis of heat transfer, thermal stress and dislocation density during resistively Czochralski growth of germanium single crysta. J. Cryst. Growth 2018, 483, 125-133. [CrossRef]

6. Sabanskis, A.; Bergfelds, K.; Muiznieks, A.; Schröck, T.; Krauze, A. Crystal shape 2D modeling for transient CZ silicon crystal growth. J. Crystal Growth 2013, 377, 9-16. [CrossRef]

7. Abrosimov, N.; Czupalla, M.; Dropka, N.; Fischer, J.; Gybin, O.; Irmscher, K.; Janicskó-Csáthy, J.; Juda, U.; Kayser, S.; Miller, W.; et al. Technology development of high purity germanium crystals for radiation detectors. J. Cryst. Growth 2019, in press. [CrossRef]

8. Abrosimov, N.V.; Lüdge, A.; Riemann, H.; Schröder, W. Lateral photovoltage scanning (LPS) method for the visualization of the solid-liquid interface of $\mathrm{Si}_{1-x} \mathrm{Ge}_{x}$ single crystals. J. Cryst. Growth 2002, 237-239, 356-360. [CrossRef]

9. Lüdge, A.; Riemann, H. Doping inhomogeneities in silicon crystals detected by the lateral photovoltage scanning (LPS) method. Inst. Phys. Conf. Ser. 1998, 160, 145-148.

10. Kayser, S.; Lüdge, A.; Böttcher, K. Computational simulations of the of lateral-photovoltage-scanning-Method. In Proceedings of the 8th International Scientific Colloquium: Modelling for Material Processing, Riga, Latvia, 21-22 September 2017; pp. 149-154. [CrossRef]

11. Morin, F.J.; Maita, J.P. Conductivity and hall effect in the intrinsic range of germanium. Phys. Rev. 1954, 94, 1525-1529. [CrossRef]

12. Geuzaine, C.; Remacle, J.-F. Gmsh: A three-dimensional finite element mesh generator with built-in pre- and post-processing facilities. Int. J. Num. Meth. Eng. 2009, 79, 1309-1331. [CrossRef]

13. Hurle, D.T.J. Analytical representation of the shape of the meniscus in Czochralski growth. J. Cryst. Growth 1983, 63, 13-17. [CrossRef]

14. Den Bogaert, N.V.; Dupret, F. Dynamic global simulation of the Czochralski process, I. Principles of the method. J. Cryst. Growth 1997, 171, 65-76. [CrossRef]

15. Lambropoulos, J.C. The isotropic assumption during the Czochralski growth of single semiconductors crystals. J. Cryst. Growth 1987, 84, 349-358. [CrossRef]

16. Rhim, W.-K.; Ishikawa, T. Thermophysical properties of molten germanium measured by a high-temperature electrostatic levitator. Int. J. Thermophys. 2000, 21, 429-443. [CrossRef]

17. Chathoth, S.M.; Damaschke, B.; Samwer, K.; Schneider, S. Thermophysical properties of Si, Ge, and Si-Ge alloy melts measured under microgravity. Appl. Phys. Lett. 2008, 93, 071902. [CrossRef]

18. Nishi, T.; Shibata, H.; Ohta, H. Thermal diffusivities and conductivities of molten germanium and silicon. Mater. Trans. 2003, 44, 2369-2374. [CrossRef]

19. Takasuka, E.; Tokizaki, E.; Terashima, K.; Kimura, S. Emissivity of liquid germanium in visible and near infrared region. J. Appl. Phys. 1997, 85, 2590-2595. [CrossRef]

20. Skinner, L.; Barnes, A.C. An oscillating coil system for contactless electrical conductivity measurements of aerodynamically levitated melts. Rev. Sci. Instrum. 2006, 77, 123904. [CrossRef]

21. Sato, Y.; Nishizuka, T.; Tachikawa, T.; Hoshi, M.; Yamamura, T.; Yamamura, T. Viscosity and density of molten germanium. High Temp. High Press. 2000, 32, 252-260. [CrossRef]

22. Artemyev, V.K.; Folomeev, V.I.; Ginkin, V.P.; Kartavykh, A.V.; Mil'vidskii, M.G.; Rakov, V.V. The mechanism of Marangoni convection influence on dopant distribution in Ge space-grown single crystals. J. Cryst. Growth 2001, 223, 29-37. [CrossRef]

23. Reeber, R.R.; Wang, K. Thermal expansion and lattice parameters of group IV semiconductors. Mater. Chem. Phys. 1996, 46, 259-264. [CrossRef]

24. Glassbrenner, C.J.; Slack, G.A. Thermal conductivity of silicon and germanium from $3 \mathrm{~K}$ to the melting point. Phys. Rev. 1964, 134, A1058-A1069. [CrossRef] 
25. Allen, F.G. Emissivity at 0.65 micron of silicon and germanium at high temperatures. J. Appl. Phys. 1957, 28, 1510-1512. [CrossRef]

26. Nikanorov, S.P.; Kardashev, B.K. Elasticity and Dislocation Inelasticity of Crystalls; Moscow Izdatel Nauka: Moscow, Russia, 1985.

27. Alexander, H.; Haasen, P. Dislocations and plastic flow in the diamond structure. Solid State Phys. 1968, 22, 27-158.

28. Haasen, P. Dislocations in semiconductors. J. Phys. Colloques 1966, 27, C3-30-C3-38. [CrossRef]

29. Dupas, C.; Zuodar, N.; Coddet, O.; Kruml, T.; Martin, J.-L. Dislocation multiplication, exhaustion and mechanical behavior for Ge single crystals. J. Phys. Condens. Matter 2002, 14, 12989-12995. [CrossRef]

30. Kruml, T.; Dupas, C.; Martin, J.L. A critical assessment of dislocation multiplication laws in germanium. Acta Mater. 2006, 54, 4721-4729. [CrossRef]

31. Helbig, R.; Funke, C.; Möller, H.J. Simulation of the dislocation density during multicrystalline silicon ingot growth. In Proceedings of the Wafer-Based Silicon Solar Cells and Materials Technology, EU PVSEC Proceedings, Hamburg, Germany, 5-9 September 2011; pp. 1938-1941. [CrossRef]

(C) 2019 by the authors. Licensee MDPI, Basel, Switzerland. This article is an open access article distributed under the terms and conditions of the Creative Commons Attribution (CC BY) license (http://creativecommons.org/licenses/by/4.0/). 